\title{
A Framework for Designing Novel Magnetic Tiles Capable of Complex Self-Assemblies
}

\author{
Urmi Majumder and John H. Reif \\ Department of Computer Science, \\ Duke University, Durham, NC, USA \\ urmim, reif@cs.duke.edu
}

\begin{abstract}
Self-assembly has been immensely successful in creating complex patterns at the molecular scale. However, the use of self-assembly techniques at the macroscopic level has so far been limited to the formation of simple patterns. For example, in a number of prior works, self-assembling units or tiles formed aggregates based on the polarity of magnetic pads on their sides. The complexity of the resulting assemblies was limited, however, due to the small variety of magnetic pads that were used: namely just positive or negative polarity. This paper addresses the key challenge of increasing the variety of magnetic pads for tiles, which would allow the tiles to self-assemble into more complex patterns. We introduce a barcode scheme which potentially allows for the generation of arbitrarily complex structures using magnetic self-assembly at the macro-scale. Development of a framework for designing such barcode schemes is the main contribution of the paper. We also present a physical model based on Newtonian mechanics and Maxwellian magnetics. Additionally, we present a preliminary software simulation system that models the binding of these tiles using magnetic interactions as well as external forces (e.g. wind) which provide energy to the system. Although we have not performed any physical experiments, nevertheless, we show that it is possible to use the simulation results to extract a higher level kinetic model that can be used to predict assembly yield on a larger scale and provide better insight into the dynamics of the real system.
\end{abstract}

\section{Introduction}

\subsection{Motivation}

Self-assembly is a process where small components spontaneously organize themselves into a larger structure. This phenomenon is prevalent on all scales, from molecules to galaxies. Though self-assembly is a bottom-up process not utilizing an overall central control, it is theoretically capable of constructing arbitrarily complex objects.

One of the most well-studied sub-fields of self-assembly is molecular selfassembly. However, many interesting applications of self-assembling processes can be found at a larger scale. Massively parallel self-assembling systems present 
a promising alternative to conventional manufacturing (which mostly uses sequential pick-and-place assembly). There are many examples of self-assembling systems at this scale which may be relevant to robotics and manufacturing such as self-assembled monolayers, the patterned assembly of electronic components and MEMS devices and the assembly of micro-robots and/or sensors.

In this paper, we explore magnetic self-assembly with the ultimate goal of discovering the practical limits for its use in manufacturing and computing systems. Most of the related work described below focuses on the demonstration of macro- and micro-scale self-assembly. However, this paper focuses more on the design issues relevant to the generation of more complex structures using our novel barcode scheme.

\subsection{Previous Work}

Recent work in the field of macro-scale self-assembly include development of systems based on capillary interactions among millimeter-scale components either floating at a fluid-fluid interface or suspended in an approximately iso-dense fluid medium [1-9]. In fact, this technique has been adopted commercially [10, 11]. Rothemund [12] demonstrated the use of hydrophobic and hydrophilic interactions to generate self-assemblies of moderate complexity and scale. His work was notable since it is the only work which demonstrated computational selfassembly at the macro-scale.

Magnetic Passive and Active Assemblies Magnetic assembly [13-15] is a form of macro-scale self-assembly that is directed by magnetic dipole interaction. One successful application of magnetic assembly is the spontaneous folding of elastomeric sheets, patterned with magnetic dipoles, into free standing, 3D spherical shells [16]. This technique has been shown to generate relatively simple structures, largely due to the limited nature of the magnetic interactions. This kind of self-assembly is also known as passive self-assembly since assembly takes place without external control. Here we address the key challenge of going beyond such limitations and aim to design more complex structures via magnetic assembly. To increase the complexity of magnetic assemblies, Klavins et al. [17] developed programmable units that move passively on an air-table and bind to each other upon random collisions. These programmable units have on-board processors that can change the magnetic properties of the units dynamically during assembly. Once attached, they execute local rules that determine how their internal states change and whether they should remain bound based on the theory of graph grammars [17]. This form of assembly is referred to as active assembly. However, our goal is to generate complex magnetic assemblies without the use of on-board processors (i.e. passive assembly).

Computational Molecular Self-Assemblies In 1966 Berger proved that, in theory, universal computation can be done via tiling assemblies [18]. This essentially showed that tiling assemblies can generate arbitrarily complex structures. However, these theoretical ideas were not put to practice until much later. 
In 1982, Seeman [19] proposed that DNA nano-structures can be self-assembled by using Watson-Crick complementarity and thus DNA can form the basis of programmable nano-fabrication (This was later demonstrated in the 1990s). A seminal paper by Adleman [20] in 1994 used one-dimensional DNA self-assembly to solve an instance of the Hamiltonian path problem, thus establishing the first experimental connection between DNA self-assembly and computation. This work inspired Winfree [21] to apply the theory of Wang tiles to show that twodimensional DNA self-assembly is capable of performing Turing Universal computation. This proposal was later verified experimentally with the demonstration of a Sierpinski Triangle pattern composed of DNA tiles [22].

\subsection{Our Contribution}

The goal of this paper is to develop techniques that will allow the self-assembly of complex structures at the macro-scale. This task is quite challenging, since the available binding mechanisms (using magnetic and capillary interaction) currently used at the macro-scale provide only for binary binding (e.g., positive and negative in the case of magnetic binding and hydrophobic/hydrophilic interactions in the case of capillary binding). By contrast, DNA provides a large number of specific bindings through the use of complementary pairs of DNA sequences that can hybridize selectively. Here, we mimic the techniques and principles of molecular self-assembly to build complex structures at the macroscopic level. The key challenge is then to extend the binding mechanisms at this scale to a much larger number of specific bindings, rather than just two. We achieve this by using a magnetic barcode technique described in this paper.

Our testbed is an example of a distributed system where a large number of relatively simple components interact locally to produce interesting global behavior. Square programmable tiles float passively on a forced air-table, mixed randomly by oscillating fans (simulates Brownian motion of the molecular scale). The tiles have a magnetic encoding on each of their faces. When they collide, if the facing poles are exactly complementary, the tile faces bind to each other and this process repeats to generate our desired final structure.

We discuss how our barcode scheme relates to achievable shapes and how we can optimize our tile design. We further describe how a rigid-body simulation environment can be used to model the testbed and performed very preliminary validation of the feasibility of using self-assembly of magnetic tiles (with barcoded pads) for the generation of patterned lattices using our simulation system. We conclude with a discussion on scalability issues and how we can use our simulation results to predict yields in larger scales. Since we are yet to perform physical experiments, the emphasis in this discussion is the methodology that makes it possible to extract high-level parameters from the low-level simulation environment.

\subsection{Organization of the Paper}

Section 1 introduces the main theme of the paper: using magnetic barcodes to direct tile-assembly. Section 2 presents the overall scheme, in particular the bar- 
code scheme and the set of achievable shapes. Section 2.3 discusses the various combinatorial, thermodynamic and physical optimization rules that can be applied to improve the yield of assembly. It also presents techniques from robot motion planning that can be applied as well to improve tile designs. Section 3 presents the simulation model and some preliminary results from simulating a simple two-tile system. It also discusses the feasibility of extracting a higher level kinetic model based on assembly/disassembly rates from the low level physical simulation model and includes a discussion on scaling of the system and yield optimization. Finally Section 4 concludes the paper with some future directions.

\section{Design of a Magnetic Self-Assembly System}

Self-assembly at the macro-scale can happen through a wide range of forces viz. gravitational, electrostatic, magnetic, capillary, etc. In the past, the driving force has been mostly capillary interaction [23]. An important point to note here that the choice of the driving force depends on several factors like scale and magnitude of the force, environmental compatibility and influence of the interactions on the function of the system. We have chosen to use magnetic force as the driving force for our self-assembling system mainly because magnetic interactions are insensitive to the surrounding medium and are independent of surface chemistry. Also range of magnetic forces can be engineered so as to control the long-range and short-range interactions between components. This is important because a key issue in the design of programmable self-assembly is the recognition between components, governed by the design, surface chemistry and topology of the interacting surfaces.

\subsection{The Overall Scheme}

The overall design of our system is as follows: the main component of our selfassembling system is a set of square wooden tiles. Each edge of a tile is lined with a sequence of magnetic dipoles. The latter is perpendicular to each face and can either have their north pole or south pole facing out. A typical encoding on a tile face may be $\{\mathrm{NNNS}\}$ where $\mathrm{N}$ or $\mathrm{S}$ denotes whether the north pole or the south pole of the dipole is facing out of the tile. The tiles float on a custom made air-table. A set of fans are placed around the air-table and mix the tiles. Thus all the interactions between the tiles are due to chance collisions. The idea is that if a tile face (e.g. with encoding $\{$ NNNS $\}$ ) collides with a tile face with matching encoding (i.e. $\{\mathrm{SSSN}\}$ ), they stick together, thus resulting in an assembly (Fig. $1)$.

\subsection{The Barcode Scheme and Achievable Shapes}

In the context of our magnetic self-assembly a barcode is a series of bar magnet poles that face out of the tile on any face (e.g. NSN and SNS as in Fig. 1). If we have a $n$ character long barcode on each face of every square tile in our tile 


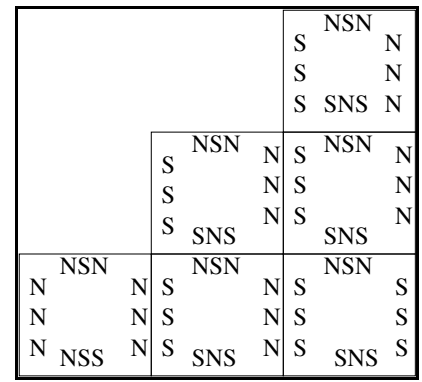

Fig. 1. A typical magnetic assembly

set then number of distinct tiles is $2^{4 n}$. However, there can be different types of assemblies ranging from uniquely addressable to homogeneous. A uniquely addressable lattice is where each tile in the assembly has a unique location. Any such lattice of size $m \times n$ calls for $m(n-1)+n(m-1)$ different barcodes. Thus, in this case, we need barcode of length $O(\log (m n))$. At the other extreme is a homogeneous lattice which calls for exactly one tile type and can be constructed with $O(1)$ length barcodes. In between these two extremes lie computational assemblies, which have been shown to be Turing Universal [24]. Here we treat each tile as a computational unit where the east and south faces are inputs of the computation while north and west are outputs of the computations, which are then used in the next step of the computation. In other words, a tile assembly model of size $n T$ simulates a Blocked Cellular Automata of size $n$ running in time $T$ [24]. For any such computation, a barcode of length $n$ generates a tile set of size $2^{2 n}$. Further the number of functions we can have is $(2)^{2^{2 n}}$.

Examples of Complex Assemblies Some examples of complex assemblies are given in Fig. 2. Each of these assemblies are based on Winfree's Tile Assembly model [24] and only uses a small number of tile types $(O(1))$, as is the characteristic of any computational assembly.

Complexity of Achievable Shapes This question was first addressed by Rothemund and Winfree [26] for computational molecular assembly. However, their results also hold for macroscopic assemblies. Suppose that $\tau$ is defined as the parameter which decides when to add a tile to a growing assembly. In particular, when the total interaction strength of a tile with its neighbors exceed $\tau$, the tile gets added to the assembly. Then the minimum number of distinct tiles required to self-assemble a $N \times N$ square decreases from $N^{2}$ to $O(\log N)$ tiles as $\tau$ is increased from 1 (noncooperative bonding) to 2 (cooperative bonding). An alternative measure is to compute the minimum number of distinct side labels used for assembling the square. It is still an open question whether both measures give asymptotically similar results. The latter will be more useful for a practical implementation of the system since, in reality, the number of distinct 

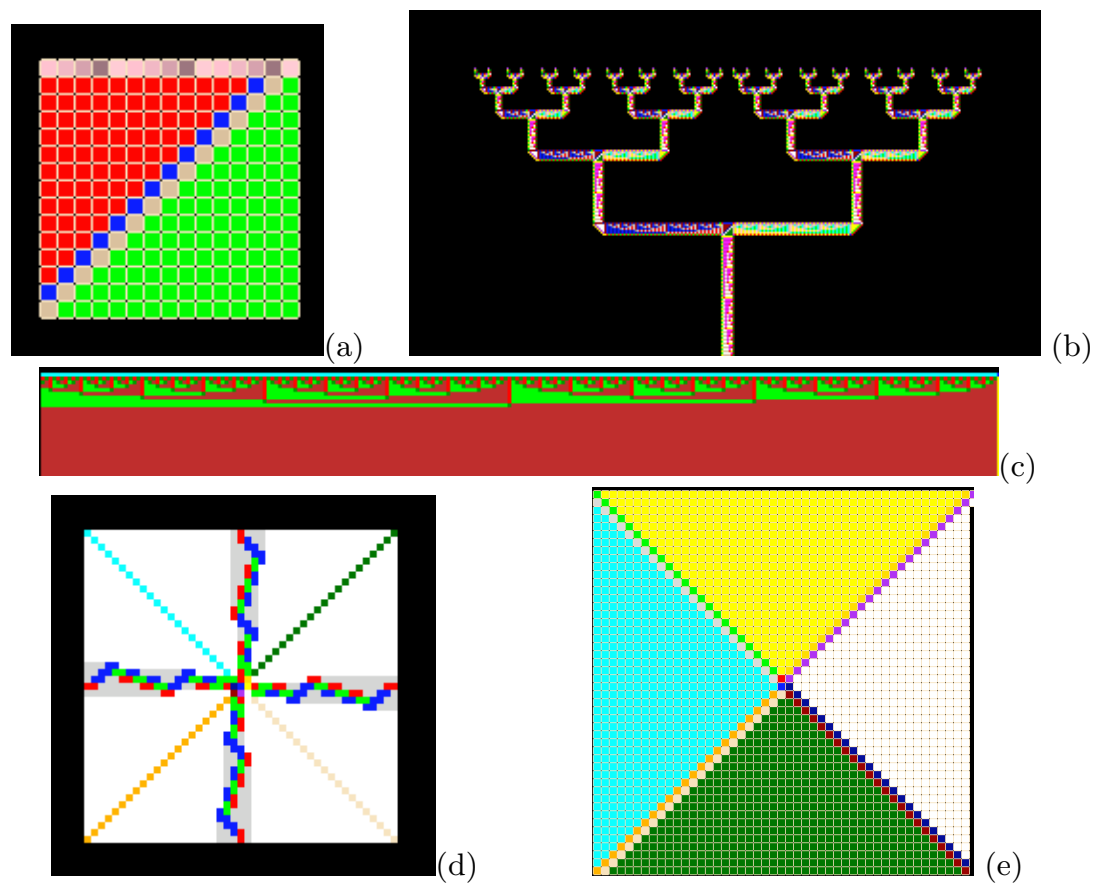

Fig. 2. Examples of Complex Assemblies: (a) Unary Square, (b) Binary Tree, (c) Binary Counter, (d) Beaver Square, (e) Spiral, made with XGROW [25].

binding interactions is limited due to imperfect specificity of binding. It should be mentioned here that Adelman et al. [27] later proved a tighter bound of $\Theta\left(\frac{\log N}{\log \log N}\right)$ for the minimum number of distinct tiles required to assemble a $N \times N$ square uniquely, by demonstrating that self-assembly can compute changes in the base representation of numbers. A further decrease was achieved by Kao et al. [28] who proved that using a sequence of $O(m)$ changes in $\tau$ (where $m$ is an arbitrarily long binary number), a general tile set of size $O(1)$ can uniquely assemble any $N \times N$ square.

For arbitrary shapes (e.g. non-squares) no such tight bounds exist as yet. However, Soloveichik et al. [29] recently showed that the minimal number of distinct tile types required to self-assemble an arbitrarily scaled structure can be bounded both above and below by the shape's Kolmogorov's complexity where Kolmogorov Complexity of a string $I$ is defined to be the length of the shortest program that computes or outputs $I$, when the program is run on some fixed reference Universal computer.

\subsection{Tile Programming}

This section describes our barcode design scheme. We will sometimes refer to the barcode on a tile face as a word. Here our goal is to design a set of words such that the energy difference between a pair of perfectly matched faces and 
a pair of partially or completely unmatched faces is maximized. Tulpan et al. [30] proposed a DNA-design algorithm based on local search approach that can be utilized for our magnetic barcode design with minimal modifications. The algorithm takes as input: the length of each code word, the number of unique codewords that need to be generated and a set of constraints that the output set must satisfy. We describe some of the constraints for magnetic tile design below:

Combinatorial Optimization Some examples of combinatorial constraints [31] are as follows:

1. The number of mismatches in a perfect alignment of two tile faces must be above an user-defined threshold. For instance if a tile face encoded as NNNN aligns up with a face encoded as SNSN then there will be two mismatches in such an alignment. Note that mismatches need not be consecutive and can be minimized using prefix codes and Hamming distance maximization.

2. The number of mismatches between a perfect alignment of one tile face encoding and the complement of another tile face encoding should also be above some threshold.

3. The situation of tile binding can be made more complicated by the presence of a slide match configuration (e.g. when a tile face bearing NNNSSNSNSN matches with another tile face bearing NNSNSNSNNS starting at the fourth location on the second face and the first one for the first tile). Hence the number of mismatches in a slide of one tile face over another must be above some threshold. The problem of slide match configuration can be handled using shift distinct codes or complementary shapes for tile faces.

4. The maximum number of consecutive matches between all slides of one tile face encoding over the other must be in an user defined range.

Thermodynamic Optimization Thermodynamic constraints are based on the free energy of a pair of tile binding. The free energy of an assembly is not just a function of the encodings, but also the number, orientation and speed of fans and number of tiles. However, any model incorporating so many free parameters will be quite complicated. Hence, for simplicity, we will assume that the sole contributor to free energy in our case is the magnetic interaction between two tile faces when they are perfectly aligned. Effects of adjacent faces (e.g. north and east) can be neglected because of shielding (Section 3.1). Some thermodynamic constraints used in the algorithm [31] are as follows:

1. The free energy of a perfect match must be below a given threshold.

2. The free energy of a code word and the complement of another code word, two words or two complements must also be in a desired range.

Eventually, the goal is to obtain a positive free energy gap between the perfect match and imperfect matches of a code word.

Since our magnetic assembly is a mechanical system, we will also take some physical factors into consideration while designing tiles. 
Physical Optimization We can minimize intra and inter-magnetic cross-talk using the following techniques [Fig. 3]:

1. Large tile to magnetic dipole size ratio (minimizes interaction between adjacent tile faces).

2. Barcodes towards the center of the face (minimizes interaction between adjacent tile faces).

3. Use of spacer sequences, thus increasing the alphabet size.

4. Use of long thin magnets, essentially minimizing the effect of one pole on another.

5. Use of magnetic shields. (a coating of soft iron on the magnets prevents coupling of flux lines between two adjacent bar magnets). An alternative method is to use Halbach array, which is a special arrangement of permanent magnets that augments the magnetic field on one side of the device while canceling the field to near zero on the other side [32]. Although in this scheme we can intensify the magnetic field at the end of the tile faces and minimize it at the other end of magnetic arrangement, the method cannot handle sideways magnetic crosstalk.

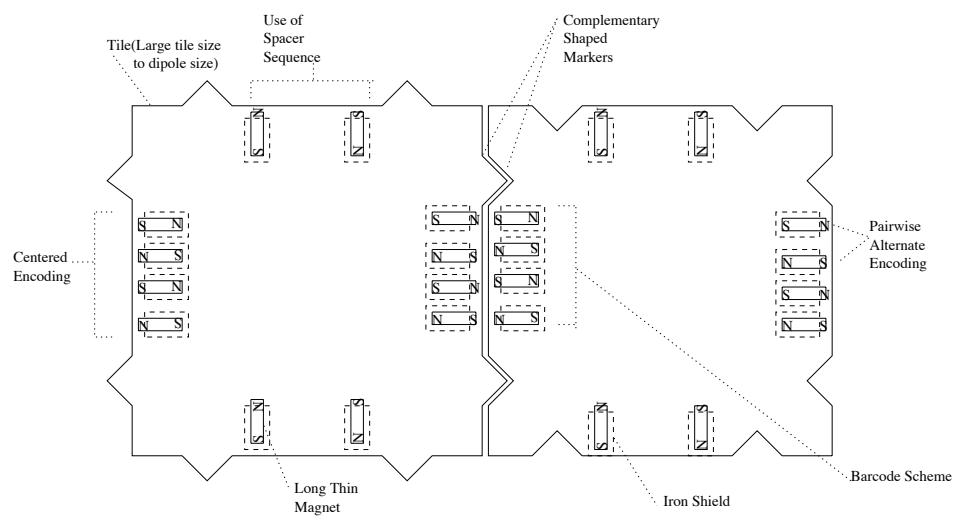

Fig. 3. Techniques for minimizing magnetic crosstalk.

\subsection{Improving Tile Designs using Motion Planning Studies}

Complementary shape matching [Fig. 3] is a useful technique in optimal tile design [33]. We can verify the "goodness" of a match using a motion planning technique called a probabilistic roadmap [34] which is mostly used to check the connectivity of a collision free path in a high-dimensional space. It can also be used to capture the extrema of a continuous function (e.g. potential energy) 
over high-dimensional space [35] since this map captures the connectivity of the low energy subset of the configuration space in the form of a network of weighted pathways. In our context, it can be used to study the potential energy landscape of a two tile assembly. Specifically it will be interesting to find out whether an energetically favorable path exists between any randomly generated configuration for the tiles and its final bound state and if it exists, compute the energy barrier. Further, it may be useful to study how the energy barrier varies with various complementary shapes and depth of the binding site.

The conformational space for a two-tile system with one fixed tile and another with some initial velocity is essentially three-dimensional $(x, y$ and $\theta)$. The energy function is based on the magnetic interaction model (See Sect. 3.1). Milestones in this configuration space are generated randomly using rejection sampling where the probability of accepting a milestone depends on the tile configuration. An edge exists between any two milestones in the configuration space if the path between them is energetically favorable and the weight is determined by the energy of the path. Once the graph is constructed there are many ways to use it. One typical query is the shortest weight path between two configurations; another query is to use it to characterize a true binding site based on the energy barrier mentioned above.

\section{Simulation of a two tile system}

\subsection{Simulation Model}

Since actual experimentation would require elaborate patterning of tiles and careful placement of fans with appropriate driving force, we evaluated our barcode scheme by simulating a two tile assembly. This section presents the physical model underlying the simulation.

Tile Motion Model The air-table provides a two-dimensional fluid environment for the tiles. As tiles traverse the testbed, they will lose kinetic energy due to friction and variations in their air cushion. In our model, we assume that our air-table surface has a very low coefficient of friction, minimizing energy losses as the tiles traverse on the air-bed.

Fan Model We use an exponentially decaying function to model the fans. Our simulation assumes that the potential energy $E_{f}$ is a function of the distance $\vec{r}$ from a tile to a fan and takes the form of $E_{f}=e^{-|r|^{2}}$. Hence the fan force can be obtained as the gradient of potential energy. Interestingly, the oscillating fans simulate the Brownian motion which is the main driving force behind diffusion and self-assembly in the molecular level.

Collision and Friction Model We assume that the coefficient of restitution between two tiles is small to allow short-range magnetic forces decide whether 
a binding event will take place or not. Our friction model is essentially an approximation of Coulomb's friction model.

Magnetic Interaction Model Since the magnets are glued to the tile surface and are shielded, intra-tile magnetic interaction is negligible. For interfacing tiles, our design ensures that only the magnets on the nearest face will have any effect on a given bar magnet.

Magnetic Dipole Approximation : We approximate our bar magnets as magnetic dipoles. We do not have any source of electric current in our system; so Maxwell's equations for magnetostatics apply in this case specifically,

$$
\begin{aligned}
\vec{\nabla} \cdot \vec{B} & =0 \\
\vec{\nabla} \times \vec{B} & =\frac{4 \pi}{c} \vec{J}
\end{aligned}
$$

where $\vec{B}$ is the flux density of the magnet and $\vec{J}$ is its electric current density. If we define $\vec{B}=\vec{\nabla} \times \vec{A}$, then for single geometries we can perform Coulomb-like integrals for $\vec{A}$ and then a multi-pole expansion of it up to the dipole term, yielding flux density $\vec{B}=\frac{3(\vec{m} \cdot \hat{r}) \hat{r}-\vec{m}}{\vec{r}^{3}}$ at a distance $\vec{r}$ due to a magnet with dipole moment $\vec{m}$. Hence the force on a dipole in an external magnetic field is $\vec{F}=\nabla(\vec{m} \cdot \vec{B})$. In particular, suppose we want to compute the force experienced by a magnet $M_{1}$ on tile $T_{1}$ due to the magnetic field $\vec{B}_{2}$ of a magnet $M_{2}$ on tile $T_{2}$ located at a distance $\vec{r}=x i+y j$. Let the dipole moment of a magnet $M_{1}$ be $\vec{m}_{1}=m_{x 1} i+m_{y 1} j$ and that of $M_{2}$ be $\vec{m}_{2}=m_{x 2} i+m_{y 2} j$. Then,

$$
\begin{aligned}
\vec{B}_{2} & =\frac{3\left(\vec{m}_{2} \cdot \hat{r}\right) \hat{r}-\vec{m}_{2}}{\vec{r}^{3}} \\
& =\frac{3\left(m_{x 2} x^{2}+m_{y 2} x y\right) i+3\left(m_{x 2} x y+m_{y 2} y^{2}\right) j}{\left(x^{2}+y^{2}\right)^{\frac{5}{2}}}-\frac{m_{x 2} i+m_{y 2} j}{\left(x^{2}+y^{2}\right)^{\frac{3}{2}}}
\end{aligned}
$$


Consequently,

$$
\begin{aligned}
\vec{F}= & \nabla\left(\vec{m}_{1} \cdot \vec{B}_{2}\right) \\
= & \nabla\left(3 \frac{\left(m_{x 1} m_{x 2} x^{2}+m_{x 1} m_{y 2} x y\right)+\left(m_{y 1} m_{x 2} x y+m_{y 1} m_{y 2} y^{2}\right)}{\left(x^{2}+y^{2}\right)^{\frac{5}{2}}}\right. \\
& \left.-\frac{\left(m_{x 1} m_{x 2}+m_{y 1} m_{y 2}\right)}{\left(x^{2}+y^{2}\right)^{\frac{3}{2}}}\right) \\
= & \left(-15 x \frac{\left(m_{x 1} m_{x 2} x^{2}+m_{x 1} m_{y 2} x y\right)+\left(m_{y 1} m_{x 2} x y+m_{y 1} m_{y 2} y^{2}\right)}{\left(x^{2}+y^{2}\right)^{\frac{7}{2}}}\right. \\
& \left.+\frac{3\left(m_{x 1} m_{x 2} 2 x+m_{x 1} m_{y 2} y\right)+3 m_{y 1} m_{x 2} y}{\left(x^{2}+y^{2}\right)^{\frac{5}{2}}}+\frac{3 x\left(m_{x 1} m_{x 2}+m_{y 1} m_{y 2}\right)}{\left(x^{2}+y^{2}\right)^{\frac{5}{2}}}\right) i \\
& +\left(-\frac{15 y\left(m_{x 1} m_{x 2} x^{2}+m_{x 1} m_{y 2} x y\right)+\left(m_{y 1} m_{x 2} x y+m_{y 1} m_{y 2} y^{2}\right)}{\left(x^{2}+y^{2}\right)^{\frac{7}{2}}}\right. \\
& \left.+\frac{3 m_{x 1} m_{y 2} x+3 m_{y 1} m_{x 2} x+3 m_{y 1} m_{y 2} 2 y}{\left(x^{2}+y^{2}\right)^{\frac{5}{2}}}+\frac{3 y\left(m_{x 1} m_{x 2}+m_{y 1} m_{y 2}\right)}{\left(x^{2}+y^{2}\right)^{\frac{5}{2}}}\right) j
\end{aligned}
$$

We can compute the dipole moment of a bar magnet of length $l$ and square cross-sectional area with $a=4 r^{2}$ as follows. With long thin magnets, we can approximate the bar magnet with a cylindrical bar magnet which can be further approximated by a solenoid which is $l$ units long, has $N$ turns each of which has area $\pi r^{2}$ sq units and current $i$. The magnetic field at the end of the coil is

$$
B_{0}=\frac{\mu_{0} N i}{2 \sqrt{l^{2}+r^{2}}}
$$

and following the analogous calculation for an electric dipole, the magnetic dipole moment

$$
|M|=\frac{2 B_{0} a l}{\sqrt{l^{2}+r^{2}}}
$$

and the direction is from the north pole to the south pole. In our case the $|M|$ is same for all magnets and can be set to some pre-defined value.

FEMM Simulations : We used Finite Elements Method Magnetics[36] to verify the effect of all the techniques described above. FEMM has a much greater capacity to represent physical situation than the rigid body simulation. The results in Fig. 4 clearly shows that magnetic shielding is an effective technique for minimizing magnetic crosstalk.

Tile Motion Model Once the individual forces have been calculated, we can model the tile motion. On the testbed, a tile's motion is described in terms of its two-dimensional linear acceleration $\left(\frac{d^{2} x}{d t^{2}}, \frac{d^{2} y}{d t^{2}}\right)$ and one-dimensional angular 


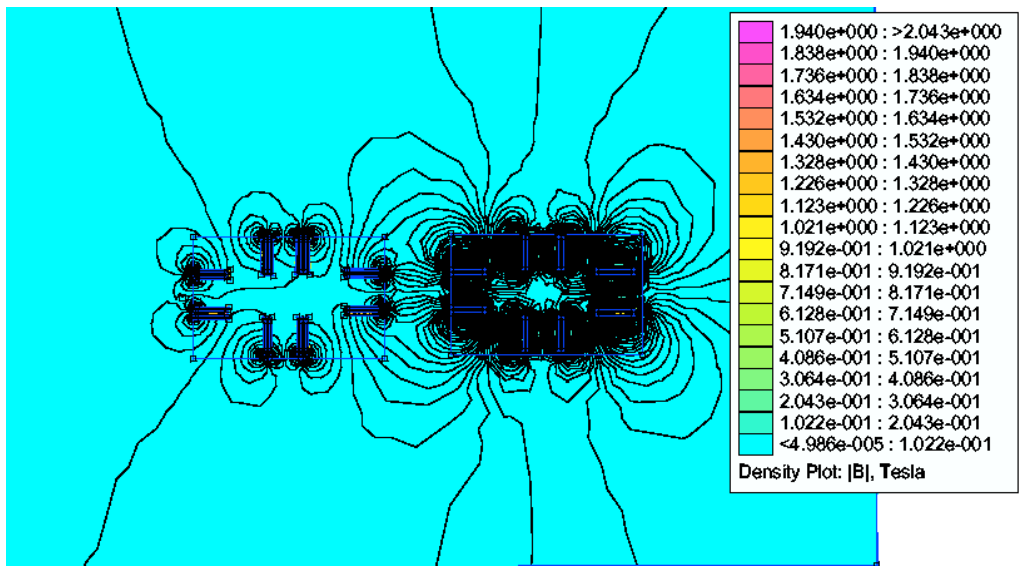

Fig. 4. FEMM Simulations showing the effect of magnetic shielding: left tile has minimum magnetic crosstalk due to magnetic shielding on its dipoles unlike the right tile with no shielding on its dipoles.

acceleration $\left(\frac{d^{2} \theta}{d t^{2}}\right)$ :

$$
\left(\begin{array}{l}
\frac{d^{2} x}{d t^{2}} \\
\frac{d^{2} y}{d t^{2}} \\
\frac{d^{2} \theta}{d t^{2}}
\end{array}\right) X=\left(\begin{array}{ccc}
-\mu & 0 & 0 \\
0 & -\mu & 0 \\
0 & 0 & -\mu
\end{array}\right)\left(\begin{array}{l}
\frac{d x}{d t} \\
\frac{d y}{d t} \\
\frac{d \theta}{d t}
\end{array}\right)+g+\frac{\overrightarrow{F_{f}}(x, y)}{m}+\frac{\overrightarrow{F_{m}}(x, y)}{m}+\frac{\vec{\tau}\left(\vec{r}, \overrightarrow{F_{m}}\right)}{I}
$$

where $\mathrm{g}$ is the acceleration due to gravity, $\mathrm{m}$ is the mass of the tile, $\mathrm{I}$ is the moment of inertia about the axis passing through its centroid perpendicular to the bed, $\mu$ is the coefficient of friction, $\overrightarrow{F_{m}}$ is the magnetic dipole force, $\overrightarrow{\tau_{m}}$ is the torque exerted on the tile by the force $\overrightarrow{F_{m}}$ acting at the magnetic point $\vec{r}$ relative to the tile's center of mass. For simplicity, we apply the fan force to the center of the tile making the torque due to the fan force equal to zero. Tiles also receive impulse forces and torques when they collide with each other or the sides of the air-table. The force and torque imparted during these events conserve linear and angular momentum but not kinetic energy, since the collisions are partially inelastic.

\subsection{Preliminary Simulation Results}

Our simulation uses the Open Dynamics Engine [37] library, which can compute trajectories of all the tiles and determine the results of the collisions. The goal of our simulation is to discover the range of the magnetic force effective for tile binding in the absence of wind energy. Here, one tile has fixed position and the other tile has an initial random position. We gave the second tile some initial 


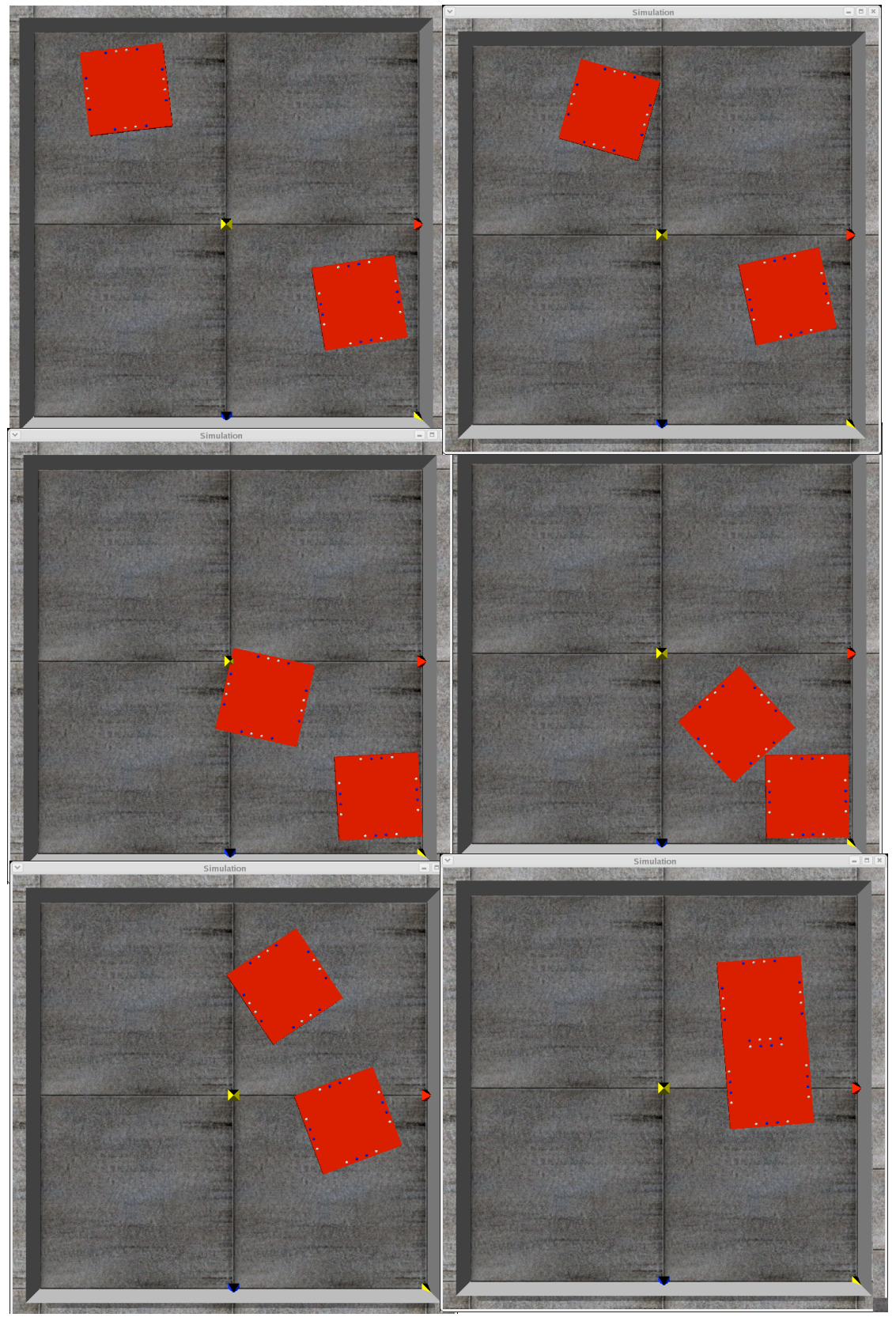

Fig. 5. (Top to Bottom, Left first, then Right) A simulation snapshot of two selfassembling square magnetic tiles (decorated with four bar magnets on each face and without complementary shapes) based on the original simulator from Klavins et al. $[17]$. 
velocity and computed the likelihood of a correct match, given the kinodynamic (position, orientation and velocity) constraints on this tile. Note that by providing the random initial velocity we are essentially simulating the exponentially decaying potential function of the wind energy source. It is important to note here that in our simulation, we call two tile faces connected if the corresponding matching dipoles are within some pre-defined threshold distance. Also, for estimating the likelihood of match in any simulation, we declare the tiles connected only when they remain connected until the end of the simulation.

Our air-bed is $2 \mathrm{~m}$ wide and $2 \mathrm{~m}$ long. The air-table has a very small coefficient of friction, specifically 0.0005 . The dimension of a tile is $43 \times 43 \times 1.3$ $\mathrm{cm}^{3}$ while that of each bar-magnet is $1 \times 1 \times 0.3 \mathrm{~cm}^{3}$. This ensures a large tile to dipole size ratio. Each tile has a mass of $100 \mathrm{~g}$. The frictional coefficient between two tile surfaces is assumed to be 0.3 while the coefficient of restitution for inter-tile collision is 0.01 . An example simulation of a two-tile assembly is shown in Figure 5. It should be remembered that the emphasis of this paper is the design framework and hence we presented only preliminary experiments.

\subsection{Interpretation of Simulation Data}

Kinetic Model The low-level simulation model based on Newtonian mechanics and Maxwellian magnetics serves as the basis for a higher level kinetic model based on on/off rates, very similar to chemical kinetics [38]. Chemical kinetics is useful for analyzing yields in large assemblies and understanding assembly dynamics without having to consider the innumerable free parameters in the low-level physical model. Although the number of tiles in our preliminary experimental setup is quite small and is not very suitable for deducing higher level model parameters, the goal here is to establish the feasibility of the process. Hence if we model tile attachment as a Poisson process, their on-rates $\lambda_{\text {on }}$ will be exponentially distributed. We, however, use the simulation data with Monte Carlo Integration to estimate $\lambda_{\text {on }}$. Similarly, the off-rate can be determined using the data on time interval between when the tiles are attached and disconnected. Figure 6 gives the probability distribution of a correct match in an assembly when the relative orientation of the two tiles is in $\left(-\frac{\pi}{2}, \frac{\pi}{2}\right)$, relative velocity is in $(0,1.3 \mathrm{~m} / \mathrm{s})$ (based on tile mass and dimensions) and relative distance between $(0,2.82 m)$ (based on the dimensions of the air-table). Unfortunately, there is no reality check on this probability distribution since we have not performed any physical experiments. Consequently this discussion is meant to present the feasibility of such an interpretation and its related benefits. As a part of future work, we intend to perform an actual validation with real data.

Scaling of the Simulation System We consider two types of scaling. In the first interpretation, we consider the relationship between the yield of assembly and the number of component tiles in the system. Intuitively, if the system is too crowded, random collisions are not possible. However, if the system is well mixed such that random collisions are possible, then, the yield of an assembly 


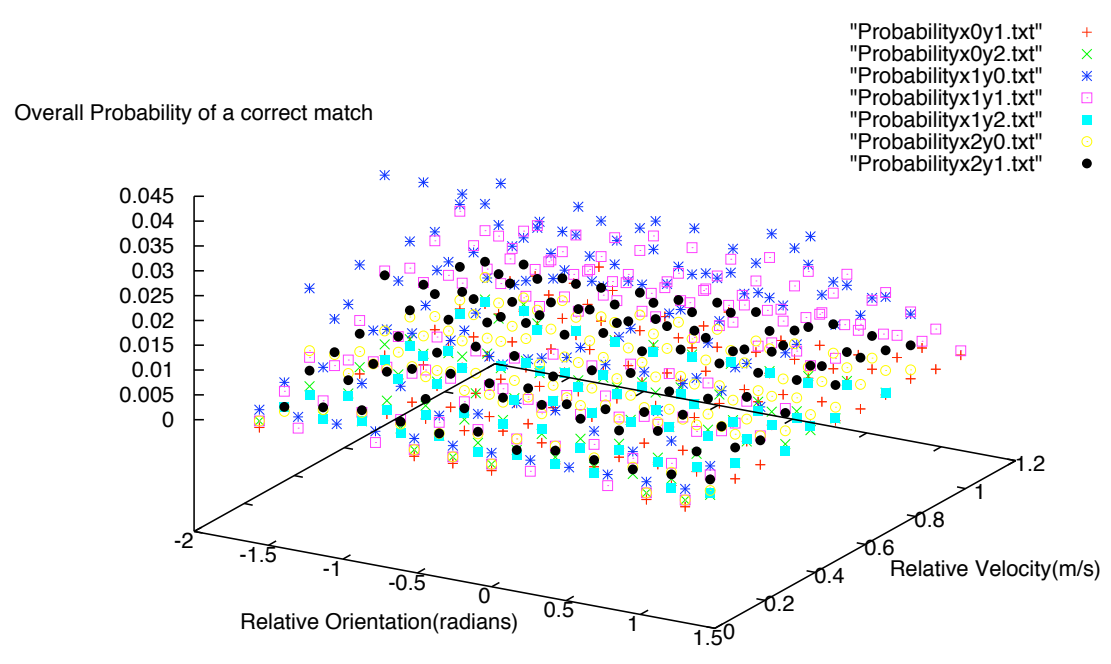

Fig. 6. Probability distribution for assembly of two tile faces for different initial positions of the moving tile (from simulation data of two tile system).

is directly proportional to the number of component magnetic tiles. We discuss more on yield optimization in Section 3.3.

Another interpretation of scale is the length scale. A major limitation to down-scaling our system is the rapid increase of the magnitude of the interactions between magnetic dipoles with the decreasing size of the particles [39]. The dipole dipole forces are attractive and scale as $d^{-4}$ where $d$ is the distance between their centers. In particular, in the nanometer scale, there is a critical length beyond which coercivity almost vanishes and the material becomes superparamagnetic [40].

Yield Optimization Since our low-level physical model leads to a model similar to a chemical kinetics model, it is possible to extract the steady state component distribution and hence use this information to design better tiles. In particular, if we interpret the system as a Markov process then we can use Master's Equation [38] to obtain the time evolution of the probability of the system to adapt one of the exponentially many configurations. We can derive the average behavior of the system using Kolmogorov's Forward Equation [41] and, thus, compute the expected number of tiles of each type in the steady state. Based on the Markov Chain interpretation it is also possible to construct a linear program in order to obtain the probabilities that would maximize the yield subject to the rate constraints, as was done by Klavins et al. [42] for active magnetic assembly. However, our system is essentially passive, hence the best we can do is to use 
these values to make small changes in the parameter space and alter the effective on and off rates and hence make incremental improvements to our yield.

\section{Future Directions}

One of the immediate goals is to extend the simulation model to a multi-tile system with fans. However, the significance of demonstration of an actual magnetic assembly cannot be undermined. Hence, one possible future direction will be the actual demonstration of the assembly and then a comparison of the experimental and simulation results, particularly the yield and the size of assembly. Another possible direction is to study the potential of a magnetic self-assembling system in three dimensions. The situation becomes more complicated in 3D due to the increase in the degrees of freedom. We would also like to study our encoding technique in a more general manner so that it can be applied to any macro and micro-scale self-assembling system. For instance, one possible direction can be the study of complex self-assembly using the capillary interaction of MEMS tiles patterned with wetting codes. Nonetheless, as an enabling technique, our hope is

that this assembly approach will be applicable to generic tiles for the generation of arbitrary complex macro-scale systems.

\section{References}

1. Bowden, N.B., Terfort, A., Carbeck, J., Whitesides, G. Science 276 (1997) 233-235

2. Bowden, N.B., Oliver, S., Whitesides, G. Journal of Phys. Chem 104 (2000) 27142724

3. Jackman, R., Brittain, S.T., Adams, A., Prentiss, M., Whitesides, G. Science 280 (1998) 2089-2091

4. Clark, T.D., Tien, J., Duffy, D.C., Paul, K., Whitesides, G. J. Am Chem. Soc. 123 (2001) 7677-7682

5. Oliver, S.R.J., N, N.B., Whitesides, G.M. J. Colloid Interface Sci 224 (2000) 425-428

6. Bowden, N., Choi, I.S., Grzybowski, B., Whitesides, G.M. J Am. Chem 121 (1999) $5373-5391$

7. Grzybowski, B., Bowden, N., Arias, F., Yang, H., Whitesides, G. J. Phys. Chem 105 (2001) 404-412

8. Syms, R.R.A., Yeatman, E.M. Electronics Lett. 29 662-664

9. Harsh, K.F., Bright, V.M., Lee, Y.C. Sens Actuators A 77 (1999) 237-244

10. Yeh, H.J.J., Smith, J.S. IEEE Photon Technol Lett 6 (1994) 706-708

11. Srinivasan, U., Liepmann, D., Howe, R.T. J. Microelectromech. Syst. 10 (2001) $17-24$

12. Rothemund, P.W.K.: Using lateral capillary forces to compute by self-assembly. PNAS (2000)

13. Gryzbowski, B., Whitesides, G.M. Nature 405 (2000) 1033-1036

14. Gryzbowski, B., Whitesides, G.M. Science 296 (2002) 718-721

15. Grzybowski, B., Jiang, X., Stone, H.A., Whitesides, G.M. Phy. Rev. E 64(111603) (2001) 1-12 
16. Boncheva, M., Andreev, S.A., Mahadevan, L., Winkleman, A., Reichman, D.R., Prentiss, M.G., Whitesides, S., Whitesides, G. PNAS 102 (2005) 3924-3929

17. Bishop, J., Burden, S., Klavins, E., Kreisberg, R., Malone, W., Napp, N., Nguyen, T.: Self-organizing programmable parts. In: Intl. Conf. on Intelligent Robots and Systems. (2005)

18. Berger, R.: The undecidability of the domino problem. Memoirs of the American Mathematical Society 66 (1966)

19. Seeman, N.C.: Nucleic acid junctions and lattices. Journal of Theor. Biology 99 (1982) 237-247

20. Adleman, L.M. Science 266 (1994) 1021-1024

21. Winfree, E. In: DNA Based Computers. (1996) 199-221

22. Rothemund, P.W.K., Papadakis, N., Winfree, E. PLoS Biology 2(12) (December 2004)

23. Whitesides, G., Boncheva, M. PNAS 99 (2002) 4769-4774

24. Winfree, E.: Algorithmic Self-Assembly of DNA. PhD thesis, California Institute of Technology (1998)

25. Winfree, E.: Simulation of computing by self-assembly. Technical Report 1998.22, Caltech (1998)

26. Rothemund, P., Winfree, E. In: STOC, ACM Press (2000) 459-468

27. Adleman, L., Cheng, Q., Goel, A., Huang, M. In: STOC, ACM Press (2001) 740748

28. Kao, M., Schweller, R. In: SODA, ACM Press (2006)

29. Soloveichik, D., Winfree, E. In: DNA Based Computers 10. LNCS (2005)

30. Tulpan, D., Hoos, H. Can. Conf. on AI, LNCS 2671 (2003) 418-433

31. Tulpan, D., Andronescu, M., Chang, S.B., Shortreed, M.R., Condon, A., Hoos, H., Smith, L.M. NAR 33(15) (2005) 4951-4964

32. J.C.Mallinson, H.Shute, Wilton, D.: One-sided fluxes in planar, cylindrical and spherical magnetized structures. IEEE Transactions on Magnetics 36(2) (March 2000)

33. Fang, J., Liang, S., Wang, K., Xiong, X., Bohringer, K.: Self-assembly of flat micro components by capillary forces and shape recognition. In: FNANO. (2005)

34. Kavraki, L., Svetska, P., Latombe, J., Overmars, M. IEEE Trans. Rob. Autom. 12(4) (1996) 566-580

35. Apaydin, M., Singh, A., Brutlag, D., Latombe, J. In: ICRA. (2001)

36. : FEMM:Finite Element Method Magnetics.

37. : http://ode.org

38. Gillespie, D. J. Phys. Chem. 81 (1977) 2340-2361

39. Gryzbowski, B., Whitesides, G. J. Phys. Chem 106 (2002) 1188-1194

40. Hu, R.L., soh, A., Ni, Y. J. Phys D: Appl Phys 39 (2006) 1987-1992

41. Strook, D.: An Introduction to Markov Processes. Springer-Verlag (2005)

42. Klavins, E., Burden, S., Napp, N.: Optimal rules for programmed stochastic selfassembly. In: RRS. (2006) 